Original Article

\title{
POTENTIAL DRUG-DRUG INTERACTIONS IN HEART FAILURE PATIENTS
}

\section{KALOYAN GEORGIEV ${ }^{*}$, NADEZHDA HVARCHANOVA², MARIETA GEORGIEVA², BRANIMIR KANAZIREV}

${ }^{1}$ Department of Pharmaceutical Technologies, Faculty of Pharmacy, Medical University of Varna, Bulgaria, ${ }^{2}$ Department of Pharmacology, Toxicology and Pharmacotherapy, Faculty of Pharmacy, Medical University of Varna, Bulgaria, ${ }^{3}$ Department of Internal Medicine, UMHAT

"St. Marina", Faculty of Medicine, Medical University of Varna, Bulgaria

Email: kalgeorgiev@hotmail.com

Received: 12 Apr 2019 Revised and Accepted: 25 Jul 2019

\section{ABSTRACT}

Objective: The aim of the present study was to assess the prevalence, risk rating and the severity of hazardous pDDIs (potential drug-drug interactions) in the prescribed pharmacotherapy in the hospital discharged heart failure (HF) patients, primarily with co-administered drugs with narrow therapeutic index (statins, anticoagulants, antithrombotic drugs).

Methods: The prescriptions of chronic heart failure patients for one year (January-December 2014) were analyzed for pDDIs through Lexi-interact ${ }^{\circledR}$ software. DDIs belonging to the categories D (Consider therapy modification) and X (Avoid combination) and/or severity of drug interaction-major, were selected for the study.

Results: After reviewing the medical records of 985 patients, 239 patients were selected based on the criteria mentioned above. The average number of prescription drugs at hospital discharge was 7.27 medications $( \pm 1.84$ SD) per patient. The total number of pDDIs was 1483 or approximately $6.2( \pm 3.89 \mathrm{SD})$ pDDIs per patient. With respect to the risk rating, in categories D and X were detected $76(5.12 \%)$ and $2(0.13 \%)$ pDDI, respectively. The major pDDIs were $108(7.28 \%)$

Conclusion: HF patients are at high risk of pDDIs. Screening of prescriptions for pDDIs and monitoring of pharmacotherapy in terms of response and associated adverse drug events will contribute to patient safety.

Keywords: Heart failure (HF), Drug-drug interactions (DDI), Anticoagulants, Statins, Antithrombotic drugs

(C) 2019 The Authors. Published by Innovare Academic Sciences Pvt Ltd. This is an open-access article under the CC BY license (http://creativecommons.org/licenses/by/4.0// DOI: http://dx.doi.org/10.22159/ijpps.2019v11i9.33585

\section{INTRODUCTION}

Heart failure (HF) affects more than 20 million people worldwide, with a $6-10 \%$ prevalence of people over $65 \mathrm{y}$ of age [1]. HF is the end stage of cardiac disease and is accompanied by several co-morbid conditions with the most common being hypertension, ischemic heart disease, hyperlipidemia, anemia, diabetes, arthritis, chronic kidney disease, chronic obstructive pulmonary disease (COPD), atrial fibrillation (AF), and Alzheimer's disease/dementia [2]. Therefore, the therapeutic regimens for the treatment of HF are very complex, including many pharmacological groups, and thus leading to polypharmacy, with great potential of drug-drug interactions (DDIs) [3-5]. There is no consensus on the definition of "polypharmacy", but in order to facilitate and reduce the degree of confusion, we will apply the most commonly used definition in the scientific literature, namely, the use of five or more drugs simultaneously [6]. "Polypharmacy" often leads to an increased risk of drug-related adverse reactions [7]. The older population is most affected by polypharmacy and its consequences. Increasing the prevalence of age-related chronic illnesses is accompanied by increased drug intake, as in the case with patients with HF.

In general, drug interactions can be considered at two levels: pharmacokinetic (PK) and pharmacodynamic (PD). PK interactions are those that occur in the processes of absorption, distribution, metabolism or drug excretion, as the latter two processes are seen as elimination processes. Most of these processes are carried out with the help of protein molecules-transporters (influx or efflux), plasma proteins, cytochrome enzymes etc., where through competitive interactions or modulation of their function, the most clinically relevant drug interactions occur $[8,9]$. Due to the remarkable individual variability in these processes, interactions can be expected, but their rate cannot be predicted easily. Such interactions may result in a change in the concentration of the drug at the site of action with subsequent toxicity or reduced efficacy. PD drug interactions occur when two or more drugs have mechanisms of action that affect the same physiological process. Influencing the physiological response of these two drugs may be in the same direction and then is referred to as synergism, and may also be in the opposite direction, and then the designation is antagonism [10]

The main purpose of the present study was to assess the prevalence risk rating and severity of hazardous pDDIs in the prescribed pharmacotherapy, primarily with co-administered drugs with narrow therapeutic index (statins, anticoagulants, antithrombotic drugs) of the discharged HF patients, to evaluate their clinical relevance, and to describe their possible mechanism of interaction.

\section{MATERIALS AND METHODS}

The medical records of 985 patients for a one-year period (JanuaryDecember 2014) at "St. Marina" University Hospital in Varna, Bulgaria were retrospectively analyzed for potential DDIs at hospital discharge using a computerized drug interaction program-Lexicomp ${ }^{\circledR}$, Lexiinteract (Wolters Kluwer, Hudson, $\mathrm{OH}$ ) [11]. The main inclusion criteria in the study were: 1) the diagnosis of HF with 2-4 class of NYHA; 2) receiving standard treatment (e. g. ACE-inhibitors or AT1blockers and/or beta-blockers, and/or diuretics, etc.); 3) concomitant use of medications threatened by pDDIs, due to a narrow safety margin, such as statins, anticoagulants (acenocoumarol or NOACs) and antithrombotic drugs (clopidogrel, prasugrel or ticagrelor). The main measure criteria were the detection of pDDIs falling into risk rating categories D (Consider therapy modification) and X (Avoid combination), or based on the severity of the drug interaction-major.

\section{Statistical analysis}

The results were presented as mean \pm standard deviation (SD) and ordinal and nominal data were shown as number (n) and percentage (\%). The statistical analysis has been done by using Excel 2016 and SigmaPlot 11.0 Software. $\mathrm{p}<0.05$ was selected as the level of statistical significance.

The Committee on Research Ethics at the Medical University "Paraskev Stoyanov" Varna/Bulgaria approved the following study (Protocol number 84/27.06.2019). The completely patient information was kept in privacy and was not available in public. 


\section{RESULTS}

After reviewing the medical records of 985 patients who have passed through the Cardiology Clinic at St. Marina University
Hospital in Varna, Bulgaria, 239 patients were selected, based on the criteria mentioned above, and the risk of drug interactions was checked with the indicated software. The workflow of the study is presented in fig. 1.

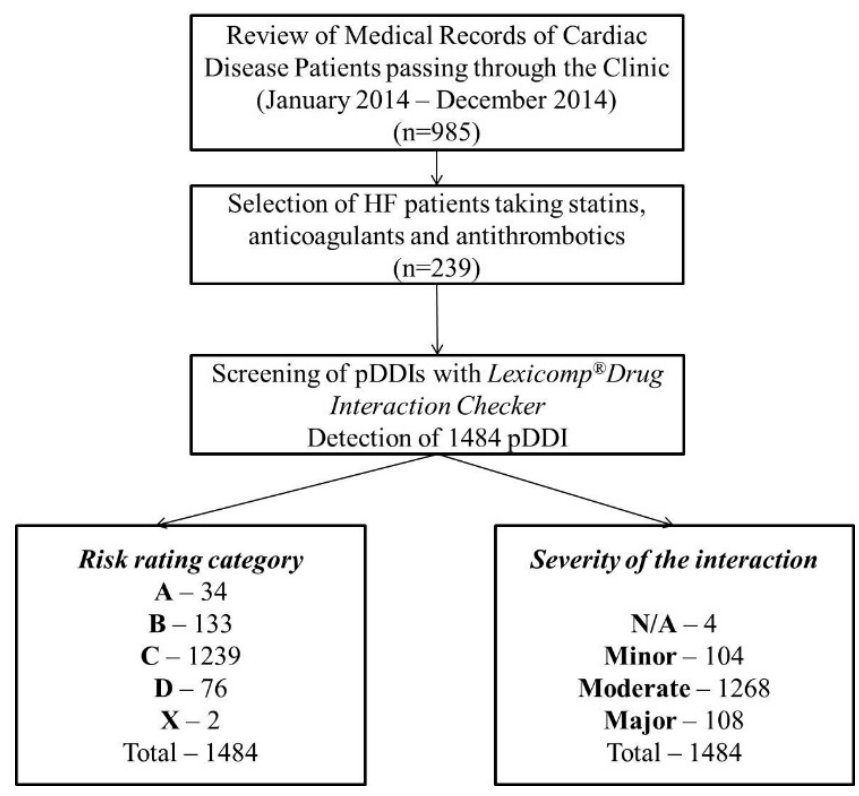

Fig. 1: Workflow of the study

The demographic characteristics of the selected patients showed almost identical gender distribution, men vs women 1:1.3. According to age, the majority of patients were in the elderly group (>60)-199 (83.3\%). The average age was $70.34( \pm 10.31 \mathrm{SD})$. The range of prescribed drugs was between four and thirteen, with an average at hospital discharge-7.27 medications $( \pm 1.84 \mathrm{SD})$ per patient. Regarding the stage of the disease, the majority of patients were in the third stage of HF (NYHA)-222 (92.9 $\%)$. All of the patients were with hypertension, the other accompanying diseases included atrial fibrillation (41\%), diabetes mellitus (41\%), and anemia (40.6\%). Reduced kidney function (eGFR $<60 \mathrm{ml} / \mathrm{min} / \mathrm{m}^{2}$ ) was detected in 87 (36.1\%), while kidney failure (eGFR $\left.<30 \mathrm{ml} / \mathrm{min} / \mathrm{m}^{2}\right)$-in $23(9.5 \%)$ of the patients (table 1 ).

Table 1: General patient characteristics selected for the study

\begin{tabular}{ll}
\hline Patients characteristics & Number, frequency (\%) \\
\hline Gender & $104(43.5 \%)$ \\
Male & $135(56.5 \%)$ \\
Female & $2(0.8 \%)$ \\
Age & $38(15.9 \%)$ \\
440 & $199(83.3 \%)$ \\
$>60$ & $70.34( \pm 10.31 \mathrm{SD})$ \\
Average age & $69.58( \pm 10.66 \mathrm{SD})$ \\
Average age male & $70.93( \pm 10.04 \mathrm{SD})$ \\
Average age female & \\
Number of prescribed drugs & $11(4.6 \%)$ \\
$<5$ & $129(54 \%)$ \\
$5-7$ & $99(41.4 \%)$ \\
$>7$ & $4-13$ \\
Range & 7.27 \\
Average & \\
Stage of heart failure (NYHA) & \\
2 & $15(6.28 \%)$ \\
3 & $222(92.9 \%)$ \\
4 & $2(0.8 \%)$ \\
Main co-morbid condition & $239(100 \%)$ \\
Hypertension & $99(41 \%)$ \\
Atrial fibrillation & $98(41 \%)$ \\
Diabetes mellitus & $97(40.6 \%)$ \\
Anemia & \\
Kidney function & $87(36.1 \%)$ \\
eGFR<60 ml/min/m m $^{2}$ & $23(9.5 \%)$ \\
eGFR<30 ml/min/m ${ }^{2}$ & \\
\hline
\end{tabular}

DDIs Detected by Lexicomp® 
The total number of pDDIs was 1484 or approximately $6.2( \pm 3.89$ SD) pDDIs per one person. According to the risk rating of the software, DDIs were classified as category A (No known interactions), B (No action needed), C (Monitor therapy), D (Consider therapy modification) and $\mathrm{X}$ (Avoid combination). The most common drug interactions with respect to the risk rating were in group C-587, requiring careful monitoring of patients. In the risk categories $\mathrm{D}$ and $\mathrm{X}$ there were $76(5.12 \%)$ and $2(0.13 \%)$ pDDIs, respectively. The software also classified drug interactions based on the severity of the interaction as major, moderate, minor, and not classified. Based on this classification, the main interactions were in the moderate group-598, while major DDIs were noted in 108 patients.

\section{DISCUSSION}

A total of 199 out of the 239 selected patients (83.3\%) in the study were elderly, and $98 \%$ of them received more than 5 drugs (polypharmacy) and thus gave us the reason to assign these patients as high-risk patients in terms of hazardous pDDIs and their therapy needed higher alertness $[3,6,12]$. In addition, 36.1 percent of patients had chronic kidney disease and 9.5 percent were with chronic renal failure, another contributing factor to the increased risk of pDDIs.

As mentioned above, the most common drug interactions were in the risk category $\mathrm{C}$, which required careful monitoring of patients. A total of 76 out of the 1484 interactions analyzed, were in risk category D (5.12 $\%)$ and their recommendations were, if possible, to consider therapy modification, and two were in category X (0.13\%)-avoid combination. Based on severity, the software detected 108 major interactions. In table 2 are presented the most common pDDIs in HF patients associated with co-prescribed narrow safety margin medications. Drugs with a narrow therapeutic index accounted for nearly $60 \%$ (46/76) of the pDDIs in risk category D. The examples and the supposed interaction mechanisms are discussed in more detail below.

Table 2: pDDIs in HF patients associated with concomitant prescribed narrow safety margin drugs

\begin{tabular}{|c|c|c|c|c|}
\hline pDDI & $\begin{array}{l}\text { Severity/Risk } \\
\text { category }\end{array}$ & $\begin{array}{l}\text { Frequency (total } \\
1484 \text { pDDI) }\end{array}$ & $\begin{array}{l}\text { Possible mechanism of } \\
\text { DDI }\end{array}$ & Literature \\
\hline \multicolumn{5}{|c|}{$\begin{array}{l}\text { The 3-hydroxy-3-methylglutaryl-coenzyme A (HMG-CoA) } \\
\text { reductase inhibitors (statins) }\end{array}$} \\
\hline Statin+1,4-DHP-CCB & Major/D & $14(0.9 \%)$ & CYP3A4 & {$[13-15]$} \\
\hline Statin+Colchicine & Major/D & $1(0.07 \%)$ & CYP3A4/OAT*/PD** & [16-19] \\
\hline Statin+Fenofibrate & Major/C & $12(0.8 \%)$ & $\mathrm{PD}$ & {$[20]$} \\
\hline \multicolumn{5}{|l|}{ Anticoagulants } \\
\hline Acenocoumarol+Allopurinol & Moderate/D & $4(0.3 \%)$ & CYP2C9 & {$[21]$} \\
\hline Acenocoumarol+SMZ/TMP & Major/D & $1(0.07 \%)$ & CYP2C9, $\mathrm{PPB}^{* * *} / \mathrm{PD}$ & {$[22]$} \\
\hline Acenocoumarol+Fenofibrate & Major/D & $1(0.07 \%)$ & СYP2C9 & {$[23]$} \\
\hline Acenocoumarol+Amiodarone & Major/D & $2(0.13 \%)$ & CYP2C9 & {$[23]$} \\
\hline Acenocoumarol+Thyrostatics & Moderate/D & $2(0.13 \%)$ & PD & [24] \\
\hline \multicolumn{5}{|l|}{ Non-vitamin Koral anticoagulants (NOACs) } \\
\hline Dabigatran+Amiodarone & Major/D & $1(0.07 \%)$ & $\mathrm{P}-\mathrm{gp}^{* * * *}$ & {$[25]$} \\
\hline Dabigatran+Verapamil & Major/D & $1(0.07 \%)$ & P-gp & [26] \\
\hline Dabigatran+Carvedilol & Major/D & $1(0.07 \%)$ & P-gp & [26] \\
\hline Rivaroxaban+Verapamil & Major/D & $1(0.07 \%)$ & CYP3A4, P-gp & {$[26,27]$} \\
\hline \multicolumn{5}{|l|}{ Antithrombotic drugs } \\
\hline Clopidogrel+Proton pump inhibitors & Moderate/D & $17(1.15 \%)$ & CYP2C19 & {$[28-30]$} \\
\hline
\end{tabular}

*OAT-organic anion transporter; **PD-pharmacodynamics; **PPB-plasma protein binding; ****P-gp-P-glycoprotein

The 3-hydroxy-3-methylglutaryl-coenzyme A (HMG-CoA) inhibitors (statins) display very complex ADME properties, which include uptake transporters (e. g. OATP1B1), biotransformation through cytochrome enzymes (e. g. CYP3A4 etc.) and efflux pumps (e. g. MDR1). All these processes are vulnerable and predispose to drug interactions. Thirteen of the 80 interactions in RRC D $(16 \%)$ involved the use of a statin in combination with a calcium channel antagonists from the 1,4-dihydropyridine group. The prescribed statin in combination was simvastatin and from the 1,4dihydropyridine calcium channel antagonists-amlodipine. The recommendations in the prescription information for both medicines are that the dose of simvastatin used in the combination with amlodipine should not exceed $20 \mathrm{mg}[13,14]$. The possible mechanism of interaction is not fully known, but it is assumed that it is about a competitive relationship with the CYP3A4 enzyme, as both preparations are substrates of this enzyme. Another commonly prescribed statin, a substrate of CYP3A4 as well, atorvastatin, does not indicate an interaction risk with amlodipine. In addition, the two drugs are included in a single dosage form for better patient adherence and compliance (Caduet ${ }^{\circledR}$ amlodipine/atorvastatin). A possible explanation for the different risk for the two substrates of CYP3A4 from the statin group is that simvastatin undergoes significant first-pass metabolism in the enterocytes of the gastrointestinal tract, while atorvastatin undergoes a less pronounced one. Interactions of simvastatin with CYP3A4 modulators are more pronounced compared to atorvastatin, and therefore atorvastatin seems to be safer for concomitant use with 1,4-dihydropyridine calcium channel antagonists [15]. Another major in severity and belonging to a risk category $D$ interaction involving statins, is the concomitant use with an antigout drugcolchicine. Only one such interaction was detected. Increased risk of myelotoxic effects-myopathy and varying degrees of rhabdomyolysis, are observed in patients taking statins with colchicine [16]. The possible mechanisms involved in this interaction are complex and are most likely to be at both pharmacokinetic and pharmacodynamic levels. The pharmacokinetic processes where this interaction can occur are the cytochrome enzyme-colchicine basically inhibits the activity of CYP3A4, but other cytochromes also, as well as the organic anionic transporter [17], and thus affects plasma statin levels. Pharmacodynamic mechanisms show that both groups of drugs are at high risk of developing myopathies [18]. Despite these possible PK and PD interactions, co-administration of statins and low-dose colchicine is nowadays recommended in patients with the coronary disease to stabilize the atherosclerotic plaque, due to inhibition of neutrophil function [19]. Another group, in which the combination of statins increases the risk of muscular toxicity, is the group of fibrates. The severity of the reaction is classified as major, while in terms of risk, it is in Category $\mathrm{C}$-monitoring of symptoms of muscle damage and when it is suspected or diagnosed, immediate discontinuation of both drugs is necessary. Among the fibrates, fenofibrate has shown the lowest risk of myopathy and the interaction potential with HMG-CoA-reductase inhibitors [20], and it is the only one registered and used fibrate in Bulgaria. The exact mechanism is not fully known, but it is assumed that mostly pharmacodynamic components take part.

Anticoagulants, and especially the group of vitamin $\mathrm{K}$ antagonists, pose a high risk of drug interactions and require increased attention. Drug interactions of this group include PK and PD mechanisms, such as displacement of binding with plasma proteins, induction or inhibition of cytochrome P450 isozymes, alterations in vitamin $\mathrm{K}$ 
status or contribution of hemorrhagic or thrombotic risk. Sixty-six patients were treated with acenocoumarol in the study. Ten of them showed pDDIs, category D with major severity, and were selected for discussion. The first case was interaction with allopurinol, a xanthin oxidase inhibitor, which is used in the treatment of gout. In this interaction, the ability of allopurinol to inhibit the metabolism of coumarin anticoagulants is likely to increase the risk of bleeding and needs close monitoring for increased prothrombin times (PT) [21]. The interaction of acenocoumarol with sulfamethoxazole/ trimethoprim (SMZ/TMP) is supposed to be more complicated. The result is a further increased risk of bleeding, but the mechanisms suggested include displacement from the plasma protein binding site, inhibition of metabolism (by inhibiting CYP2C9), but also affecting the intestinal flora responsible for vitamin $\mathrm{K}$ synthesis. Recommendations for using this combination are both dose reduction of coumarin anticoagulant and patient education for close monitoring of the INR (international normalized ratio) values [22]. The third and the fourth detected interactions were with the preparation of the fibrate group-fenofibrate and antiarrhythmic drug-amiodarone. The putative mechanisms of these interactions include inhibition of CYP2C9, the isozyme of P-450 primarily responsible for the conversion of (S)-warfarin to its major metabolite, (S)-7-hydroxywarfarin. Concomitant use of acenocoumarol with fenofibrate or amiodarone may result in a twofold increase in INR values; therefore, the recommendations are empirically reducing $20 \%$ of the dose of coumarin anticoagulant and careful monitoring of INR to see if more dose reduction is needed [23]. Coumarin anticoagulants show a notable effect in hyperthyroid patients compared to hypothyroid ones. The reason for this is the more pronounced metabolism of vitamin K-dependent blood clotting factors in patients with increased thyroid function. Hence, in patients receiving thyreostatics, such as methimazole and propylthiouracil, the effects of vitamin $\mathrm{K}$ antagonists can be compromised [24]. There is a need to monitor the changes in thyroid function and a need of dose adjustment of coumarin anticoagulants.

Among non-vitamin K oral anticoagulants (NOACs), dabigatran was the most commonly prescribed to the patients. Dabigatran etexilate is a direct thrombin inhibitor and it is indicated for the treatment of $\mathrm{AF}$, deep vein thrombosis (DVT) and/or pulmonary embolism (PE)
[25]. A pharmacokinetic feature of dabigatran is that it is a substrate of P-glycoprotein/ABCB1, and interactions with inhibitors, e. g. amiodarone, verapamil or carvedilol, of this efflux pump may occur. Management of this pDDI depends on the indication and the kidney function of the patient. Recommendations are in the treatment or prophylaxis of venous thromboembolism (VTE) and CrCL (creatinine clearance) $<50 \mathrm{ml} / \mathrm{min}$ or in the treatment of $\mathrm{AF}$ with $\mathrm{CrCL}<30 \mathrm{ml} / \mathrm{min}$ to avoid concurrent use of dabigatran etexilate with amiodarone, verapamil or carvedilol due to the risk of bleeding $[25,26]$. Other agents in the NOAC group are direct inhibitors of factor Xa, such as rivaroxaban and apixaban. Like dabigatran, these preparations are substrates of P-gp and, in addition, of CYP3A4 as well. One major drug interaction (RRC D) is detected, a combination of rivaroxaban with verapamil-moderate CYP3A4 inhibitor and P-gp inhibitor. Although in a large retrospective study, concomitant use of rivaroxaban with moderate inhibitors of CYP3A4 and P-gp did not significantly increase the risk of major bleeding [26], recommendations are for cautious use of these drugs, especially in patients with creatinine clearance $<80 \mathrm{ml} / \mathrm{min}$ [27].

Clopidogrel, an oral inhibitor of the platelet P2Y12 receptor for adenosine 5'-diphosphate (ADP), is often a part of dual antiplatelet therapy (DAPT) along with aspirin. The pharmacokinetic feature of clopidogrel that makes it susceptible to drug interactions is the biotransformation that it undergoes in the liver to form its active metabolite. The formation of an active metabolite is comprised of two steps and the major cytochrome, which takes part is CYP2C19 [28]. Proton pump inhibitors (PPIs), e. g. Omeprazole, esomeprazole, lansoprazole, pantoprazole and rabeprazole, are commonly combined with DAPT for reducing the incidence of serious gastrointestinal hemorrhages [29]. Proton pump inhibitors may inhibit the activity of CYP2C19 and the formation of the active metabolite of clopidogrel. The highest potential for clinically significant interaction was observed for omeprazole and esomeprazole. It was lower for lansoprazole and lowest for pantoprazole and rabeprazole [30].

Drug interactions in risk category $\mathrm{D}$, not related to the abovementioned groups, accounting for $40 \%$ of them, are presented in table 3 .

Table 3: pDDIs in HF patients associated with drugs, other than statins, anticoagulants and antithrombotic drugs

\begin{tabular}{|c|c|c|c|}
\hline pDDI & Severity/Risk category & Frequency & Possible mechanism of DDI \\
\hline Beta-blockers + Alfa2-agonists or $\mathrm{I}_{1}$-agonists & Moderate/D & $18(23.7 \%)$ & PD* \\
\hline Torasemide+NSAIDs & Major/D & $3(0.07 \%)$ & PD \\
\hline Azithromycin+Amiodarone, Sotalol & Major/D & $2(0.13 \%)$ & Increase of QTc \\
\hline Allopurinol+Lisinopril & Major/D & $2(0.13 \%)$ & Increase hypersensitivity \\
\hline Torasemide+Methotrexate & Major/D & $1(0.9 \%)$ & $\mathrm{OAT}^{* *}$ \\
\hline Methotrexate+Etoricoxib & Major/D & $1(0.9 \%)$ & OAT \\
\hline Amlodipine+Clarithromycin & Moderate/D & $1(0.9 \%)$ & CYP3A4 \\
\hline Sitagliptine+Sulfonylureas & Major/D & $1(0.9 \%)$ & $\mathrm{PD}$ \\
\hline
\end{tabular}

*PD-pharmacodynamics; ${ }^{* *} \mathrm{OAT}$-organic anion transporter

The most frequent reaction was the interaction of beta-blockers with alpha2-agonists or imidazoline $\mathrm{I}_{1}$-receptor agonists. Although the reaction belongs to risk category $\mathrm{D}$, the severity is considered moderate. The recommendations include heart rate monitoring, because of the increased risk of sinus node dysfunction or the onset of an AV block.

Two interactions fell in risk category X (avoid combination) and the both were the combination of cefuroxime with lansoprazole. This drug interaction has not been evaluated in any clinical study. Cefuroxime axetil salt is stable in lower $\mathrm{pH}$, therefore drugs, which increase $\mathrm{pH}$ of the stomach, will reduce the solubility and decrease the bioavailability [31]. The recommendation in the prescription information of cefuroxime axetil is to avoid concurrent use with proton pump inhibitors.

\section{Limitation of the study}

In the present study, a single program for the analysis of potential drug interactions was used. The reason for this is the detailed information provided by the software, the precise mechanisms of interaction (where known), the available information, the recommendations and the many available references. The other available softwares have also been tested, but in the end, we have been working with this one. Another limitation of the study was that no data was provided for these patients after their discharge from the hospital. However, a significant number of patients are included in the study in order for the conclusions to be made.

\section{CONCLUSION}

HF patients are at high risk of pDDIs. The review of patients' prescriptions for potential drug interactions should be done by using the appropriate software and/or a clinical pharmacist experienced in drug interactions. Moreover, we need to monitor the patient response and associated adverse effects, in order to discover the undescribed or unexplained interaction reactions. This is the only possible way to find and prevent significant side effects and eventually to improve the patient safety. 


\section{AUTHORS CONTRIBUTIONS}

Conceived and designed the study and wrote the manuscript: Kaloyan Georgiev. Data acquisition and analysis: Nadezhda Hvarchanova, Marieta Georgieva and Branimir Kanazirev. All authors read and approved the final manuscript.

\section{CONFLICTS OF INTERESTS}

Conflicts of Interest: none declared

\section{REFERENCES}

1. Rogers C, Bush N. Heart failure: pathophysiology, diagnosis, medical treatment guidelines, and nursing management. Nurs Clin North Am 2015;50:787-99.

2. Yancy CW, Jessup M, Bozkurt B, Butler J, Casey DE Jr, Colvin MM, et al. ACC/AHA/HFSA focused update of the 2013 ACCF/AHA guideline for the management of heart failure: a report of the American College of Cardiology/American heart association task force on clinical practice guidelines and the heart failure society of America. Circulation 2017;136:e137-e161.

3. Mastromarino V, Casenghi M, Testa M, Gabriele E, Coluccia R, Rubattu S, et al. Polypharmacy in heart failure patients. Curr Heart Fail Rep 2014;11:212-9.

4. Vittalrao AM, Thanusubramanian H, Kumari KM, Shaik AB. Pharmacotherapy of heart failure. Asian J Pharm Clin Res 2018;6:78-87.

5. Marushchak M, Krynytska I. Pharmacological treatment of osteoporosis in patients with coronary heart disease complicated by chronic heart failure. Asian J Pharm Clin Res 2019;1:443-6.

6. Gnjidic D, Hilmer SN, Blyth FM, Naganathan V, Waite L, Seibel MJ, et al. Polypharmacy cut off and outcomes: five or more medicines were used to identify community-dwelling older men at risk of different adverse outcomes. J Clin Epidemiol 2012;65:989-95.

7. Bushardt RL, Massey EB, Simpson TW, Ariail JC, Simpson KN. Polypharmacy: misleading, but manageable. Clin Interv Aging 2008;3:383-9.

8. Zhou SF. Drugs behave as substrates, inhibitors and inducers of human cytochrome P450 3A4. Curr Drug Metab 2008;9:310-22.

9. Huo X, Liu K. Renal organic anion transporters in drug-drug interactions and diseases. Eur J Pharm Sci 2018;112:8-19.

10. Roberts AG, Gibbs ME. Mechanisms and the clinical relevance of complex drug-drug interactions. Clin Pharmacol 2018;10:123-34.

11. Lexicomp $₫$ interaction analyser (Lexicomp $\AA$ Inc., Ohio, USA). Available from: http://www.uptodate.com [Last accessed on 05 Mar 2019]

12. Kadam UT, Roberts I, White S, Bednall R, Khunti K, Nilsson PM, et al. Conceptualising multiple drug use in patients with comorbidity and multimorbidity: proposal for standard definitions beyond the term polypharmacy. J Clin Epidemiol 2019;106:98-107.

13. Zocor (simvastatin) [prescribing information]. Whitehouse Station NJ: Merck and Co, Inc.; 2011.

14. Norvasc (amlodipine) [prescribing information]. New York, NY: Pfizer Inc; 2015.

15. Zhou YT, Yu LS, Zeng S, Huang YW, Xu HM, Zhou Q. Pharmacokinetic drug-drug interactions between 1,4- dihydropyridine calcium channel blockers and statins: factors were determining interaction strength and relevant clinical risk management. Ther Clin Risk Manag 2014;10:17-26.

16. Tufan A, Dede DS, Cavus S, Altintas ND, Iskit AB, Topeli A. Rhabdomyolysis in a patient treated with colchicine and atorvastatin. Ann Pharmacother 2006;40:1466-9.

17. Dvorak Z, Modriansky M, Pichard Garcia L, Balaguer P, Vilarem MJ, Ulrichova J, et al. Colchicine down-regulates cytochrome P450 2B6, 2C8, 2C9, and 3A4 in human hepatocytes by affecting their glucocorticoid receptor-mediated regulation. Mol Pharmacol 2003;64:160-9.

18. Valiyil R, Christopher Stine L. Drug-related myopathies of which the clinician should be aware. Curr Rheumatol Rep 2010;12:213-20.

19. Verma S, Eikelboom JW, Nidorf SM, Al-Omran M, Gupta N, Teoh $\mathrm{H}$, et al. Colchicine in cardiac disease: a systematic review and meta-analysis of randomized controlled trials. BMC Cardiovasc Disord 2015;15:96.

20. Neuvonen PJ, Niemi M, Backman JT. Drug interactions with lipid-lowering drugs: mechanisms and clinical relevance. Clin Pharmacol Ther 2006;80:565-81.

21. Pond SM, Graham GG, Wade DN, Sudlow G. The effects of allopurinol and clofibrate on theelimination of coumarin anticoagulants in man. Aust N Z J Med 1975;5:324-8.

22. Hale SF, Lesar TS. Interaction of vitamin $\mathrm{K}$ antagonists and trimethoprim-sulfamethoxazole: ignore at your patient's risk. Drug Metabol Drug Interact 2014;29:53-60.

23. Kim KY, Mancano MA. Fenofibrate potentiates warfarin effects. Ann Pharmacother 2003;37:212-5.

24. Howard Thompson A, Luckey A, George C, Choby BA, Self TH. Graves' disease and treatment effects on warfarin anticoagulation. Case Rep Med 2014. http://dx.doi.org/10.1155/2014/292468

25. Pradaxa (Dabigatran etexilate) [prescribing information]. Boehringer Ingelheim Pharmaceuticals Inc; 2018.

26. Chang SH, Chou IJ, Yeh YH, Chiou MJ, Wen MS, Kuo CT, et al. Association between use of non-vitamin k oral anticoagulants with and without concurrent medications and risk of major bleeding in nonvalvular atrial fibrillation. JAMA 2017;318:1250-9.

27. Xarelto (rivaroxaban) [prescribing information]. Titusville, NJ: Janssen Pharmaceuticals Inc.; 2017.

28. Kazui M, Nishiya Y, Ishizuka T, Hagihara K, Farid NA, Okazaki O, et al. Identification of the human cytochrome P450 enzymes involved in the two oxidative steps in the bioactivation of clopidogrel to its pharmacologically active metabolite. Drug Metab Dispos 2010;38:92-9.

29. Agewall S, Cattaneo M, Collet JP, Andreotti F, Lip GY, Verheugt FW, et al. Expert position paper on the use of proton pump inhibitors in patients with cardiovascular disease and antithrombotic therapy. Eur Heart J 2013;34:1708-1713, 1713a-1713b.

30. Norgard NB, Mathews KD, Wall GC. Drug-drug interaction between clopidogrel and the proton pump inhibitors. Ann Pharmacother 2009;43:1266-74.

31. Sommers De K, Van Wyk M, Moncrieff J, Schoeman HS. Influence of food and reduced gastric acidity on the bioavailability of bacampicillin and cefuroxime axetil. $\mathrm{Br} \mathrm{J}$ Clin Pharmacol 1984;18:535-9. 